\title{
PAVEMENT MARKING REFLECTIVITY EVALUATION THROUGH RADIOMETRIC CALIBRATION OF THE LEICA P40 TERRESTRIAL LASER SCANNER
}

\author{
E. Che ${ }^{1}$, M. J. Olsen ${ }^{1} *$, C. Parrish ${ }^{1}$, J. Jung ${ }^{1}$ \\ ${ }^{1}$ School of Civil and Construction Engineering, Oregon State University, 101 Kearney Hall, Corvallis, Oregon, U.S.A - (chee, \\ Michael.Olsen, Christopher.Parrish, Jaehoon.Jung)@oregonstate.edu
}

KEY WORDS: Terrestrial Laser Scanning, Mobile Laser Scanning, Radiometric Calibration, Road Markings, Lidar, Point Clouds

\begin{abstract}
:
Pavement markings serve as important traffic control devices, delineating traffic lanes and conveying regulations, guidance or warnings to roadway users. To ensure that pavement markings are clearly visible, especially at night, transportation agencies periodically assess the retroreflectivity of various categories of markings through manual approaches at discrete location. Because the radiometric information such as intensity in the lidar data cannot inherently be considered as a retroreflectivity measurement without additional processing, this study rigorously assesses the ability to determine pavement marking retroreflectivity from the Leica ScanStation P40 through radiometric calibration. For the evaluation, data were collected at a study site in Philomath, Oregon using the Leica P40, Leica Pegasus: Two mobile lidar system, and a handheld retroreflectometer as a reference. The results show that, with appropriate calibration, the lidar data can adequately assess the retroreflectivity of pavement markings. Additionally, while corrections have been proposed for range and angle of incidence, these corrections are not straightforward to apply for retroreflective materials, as will be discussed herein. While mobile lidar technology is ideal for a system-wide asset management framework, terrestrial laser scanning can be utilized for detailed investigations at sites such as intersections with highly variable wear where both can enable significant cost savings and applied for a variety of purposes simultaneously including asset management and project development.
\end{abstract}

\section{INTRODUCTION}

\subsection{Overview}

Pavement markings serve as important traffic control devices, delineating traffic lanes and conveying regulations, guidance or warnings to roadway users. To ensure that pavement markings are clearly visible, especially at night, transportation agencies periodically assess the retroreflectivity of various categories of markings in accordance with the Federal Highway Administration's Manual on Uniform Traffic Control Devices (MUTCD), Section 3A.03. A typical method of assessing retroreflectivity is through the use of a handheld retroreflectometer, designed to simulate the reflection of light from the headlights of a car. Alternately, visual nighttime inspection can be performed for a qualitative measure of marking quality. However, the former method is expensive, labour intensive, and carries safety implications, due to requiring roadside work, while the latter provides less quantifiable data. A promising alternative is to derive accurate retroreflectivity measurements from lidar data. As many state transportation agencies are already collecting mobile lidar data for a variety of other purposes (Olsen et al. 2013), including asset management and project development, significant cost savings could be enabled by using the lidar data to simultaneously assess pavement marking retroreflectivity.

However, lidar "intensity" data, a measure of the returned signal strength for each detected echo, cannot inherently be taken as a retroreflectivity measurement without additional calibration and processing because the intensity value is affected by a variety of factors, including scanning geometry (e.g., angle of incidence, range), environmental conditions, scanner settings (e.g., internal manufacturer corrections), and material properties. Various correction methods have been proposed, primarily focused on range and angle of incidence. Kashani et al. (2015) provide an overview of approaches for intensity normalization (making intensity values consistent between adjacent flight lines or scans), intensity correction (applying a correction based on parameters such as range), and radiometric calibration (performing a rigorous calibration to objects of known reflectance). Most work in this realm focuses on airborne laser scanning; however, studies are increasingly focusing on mobile and terrestrial laser scanning (MLS and TLS). While the scanning geometry (e.g., angle of incidence, range) tends to be more consistent in ALS, scanning geometry can vary greatly in MLS and TLS for many objects.

Jaakkola et al. (2008) normalized the intensity within a cross section of the road by fitting a curve such that it can correct the intensity of the points close to the road boundary. Similarly, Guan et al. (2014) assumed a Gaussian normal distribution of the intensity across the road surface to support the following road marking extraction. Zhang et al. (2016) developed an intensity correction based on a linear regression of the cosine of the scan angle rank versus intensity to improve its consistency as a relative measure for comparing road markings. Their primary focus in developing this correlation is to utilize this information for improving the road marking extraction algorithm results. Carlson et al. (2017) evaluated the use of Velodyne HDL-32 system for retroreflectivity measurements on sheeting material mounted in different orientations but unfortunately was unable to find a satisfactory correlation with that system. Olsen et al. (2018) evaluated the Leica Pegasus: Two and found good agreement with a handheld retroreflectometer, particularly when the system was operated in a dual profiler configuration.

\footnotetext{
* Corresponding author
} 
In addition to the pavement markings, Ai and Tsai (2016) performed a radiometric calibration for mobile lidar system to evaluate the condition of the traffic signs, which are also a critical type of traffic control device that is retroreflectivitive for nighttime visibility. Olsen et al. (2018b), however, tested a different mobile lidar system that could not provide adequate retroreflectivity measures on signs.

While few studies have focused on using lidar data for pavement marking retroreflectivity evaluation, several studies have utilized intensity information for road marking extraction (e.g., Guan et al. 2016, Zhang et al. 2016, Jung et al. 2019) or to serve as a reference for improved geo-referencing (e.g., Toth et al. 2008).

\subsection{Challenges and Objectives}

This study expands on prior efforts (Olsen et al. 2018) by rigorously assessing the ability to determine pavement marking retroreflectivity using the Leica ScanStation P40. Data at a study site in Philomath, Oregon, United States, were collected using a Leica Pegasus: Two mobile lidar system and a handheld retroreflectometer in addition to the P40. While MLS is a better solution for asset management applications due to its efficiency, TLS can be used for a detailed analysis at a specific site of interest (e.g., intersection or local project site).

Several challenges arise when utilizing TLS for this application. Firstly, in locations where assessments would be conducted, wear is this means that portion of the stripe will be more intact than others leading to different mechanisms of reflectance (e.g., diffused reflection on the pavement surface compared with retroreflection on the pavement marking). Hence, one needs to know the appropriate material type first in order to apply the correct model on a per point basis rather than being able to use this information to classify the point. Additionally, not all stripes are made of retroreflective materials (e.g., glass beads, retroreflective tape). Some markings are consisting solely of reflective paint. Secondly, sample spacing (point density) is highly variable with TLS and degrades with distance along the pavement surface. This leads to substantial sampling close to the scanner on the markings and sparse sampling at further distances.

To this end, the specific objectives of this paper are to:

- Evaluate potential intensity corrections and applicability to this problem domain

- Develop correlation for retroreflectivity for the Leica ScanStation P40.

- Develop a new weighting strategy for mitigating the impact of variant sample spacing based on point sampling to model the retroreflectometer footprint.

\section{DATA COLLLECTION}

The field test was performed on a two-lane road in the Philomath project site. A specific stretch ( $75 \mathrm{~m})$ of the longitudinal stripe was chosen to ensure that a wide range of reflective stripe conditions could be evaluated (Figure 1).

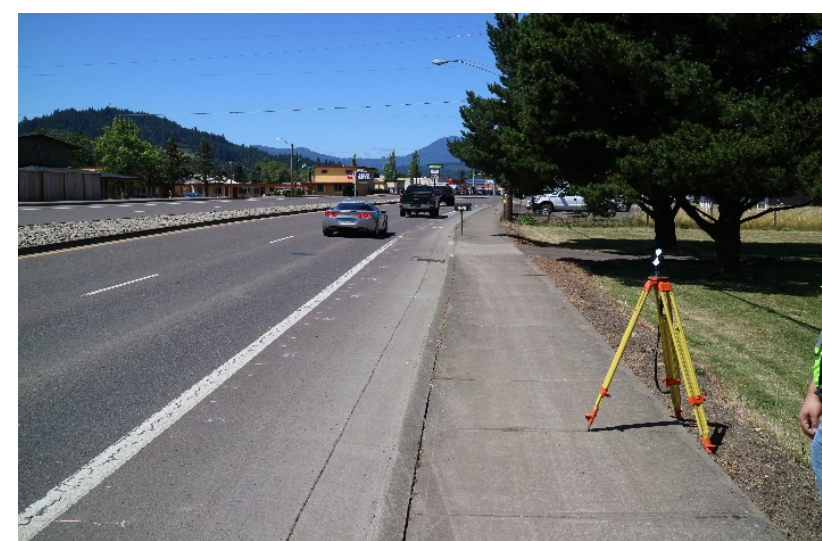

Figure 1. Photograph of the Philomath site with stripe of variable wear showing chalk markings at $0.5 \mathrm{~m}$ increments

First, we marked the stripe every $0.5 \mathrm{~m}$ along the center of the longitudinal stripe, to consistently locate a total of 155 retroreflectivity measurements using the Delta LTL-X handheld retroreflectometer (Figure 2(a)). Then, the position of each measurement point was captured with a Leica TS15 total station. A Leica Scan Station P40 terrestrial laser scanner was used to acquire a total of 9 scans spaced every $10 \mathrm{~m}$ along the stripe in the shoulder ( $\sim 2 \mathrm{~m}$ laterally from the stripe) with an angular resolution of $0.02^{\circ}$ both horizontally and vertically (Figure 2(b)). In addition, we collected MLS data using a Leica Pegasus:Two mobile laser scanner throughout the area of interest with 4 passes from each lane at a speed of $40 \mathrm{kph}$ (Figure 2(c)). Although the retroreflectometer is equipped with an internal GPS (few meter accuracy) and a GPS receiver can be mounted on the other instruments (e.g., total station, TLS, and MLS), to achieve a higher relative accuracy for developing the radiometric calibration model, all the data were registered and georeferenced via 5 ground control points set up and measured through static GNSS observations with Leica GS14 GNSS receivers.

To perform a radiometric calibration to enable the TLS to evaluate worn road markings, we purposely selected a stripe with a wide range of wear (Figure 2(b)). The fieldwork process ensured sufficient georeferencing accuracy to perform a reliable radiometric calibration, while the dense point cloud adequately represented the variation of reflectance in intensity (Figure 3).

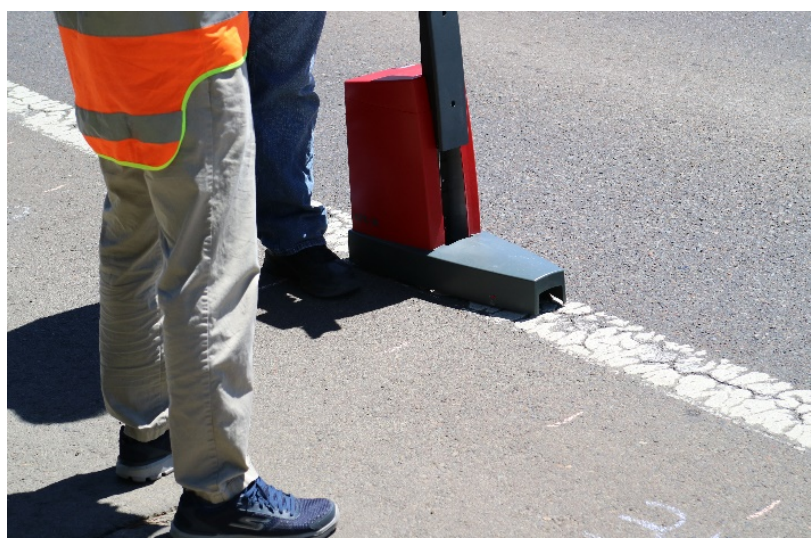

(a) 


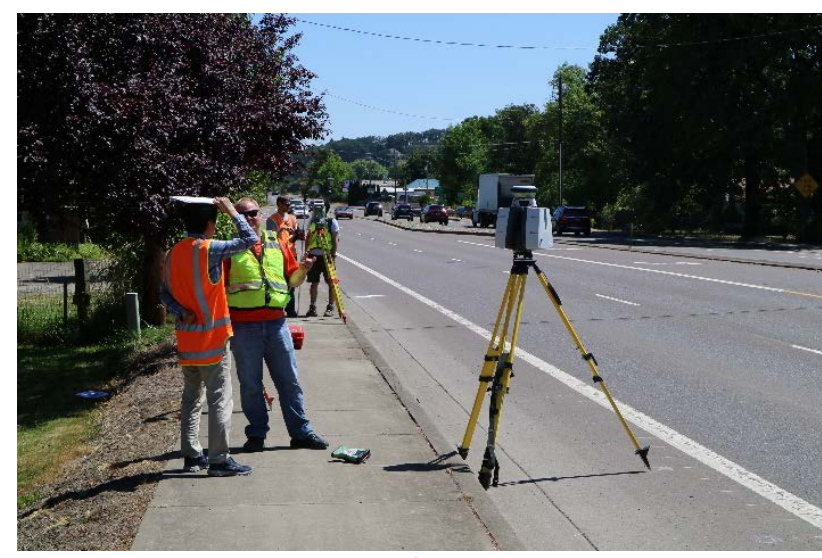

(b)

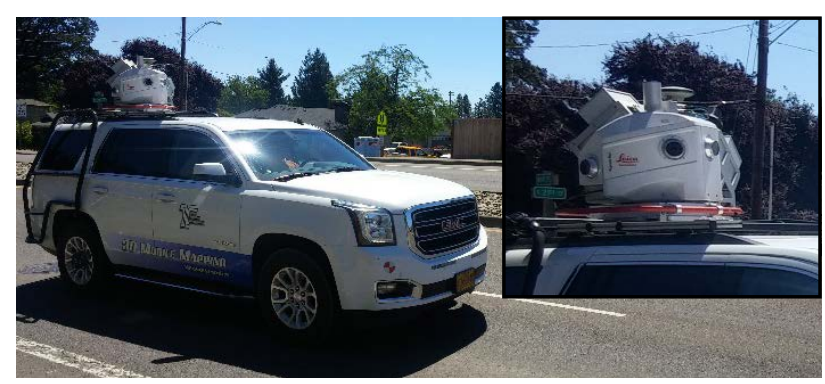

(c)

Figure 2. Examples of field data acquisition including (a) handheld retroreflectomer, (b) Leica ScanStation P40 Terrestrial Laser Scanner and Leica TS15 Total Station, and (c) Leica Pegasus Mobile laser scanner

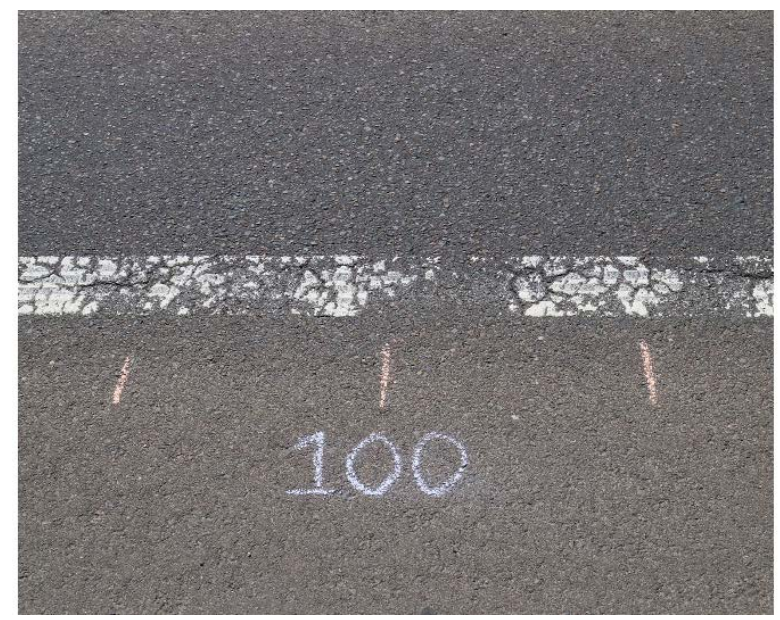

(a)

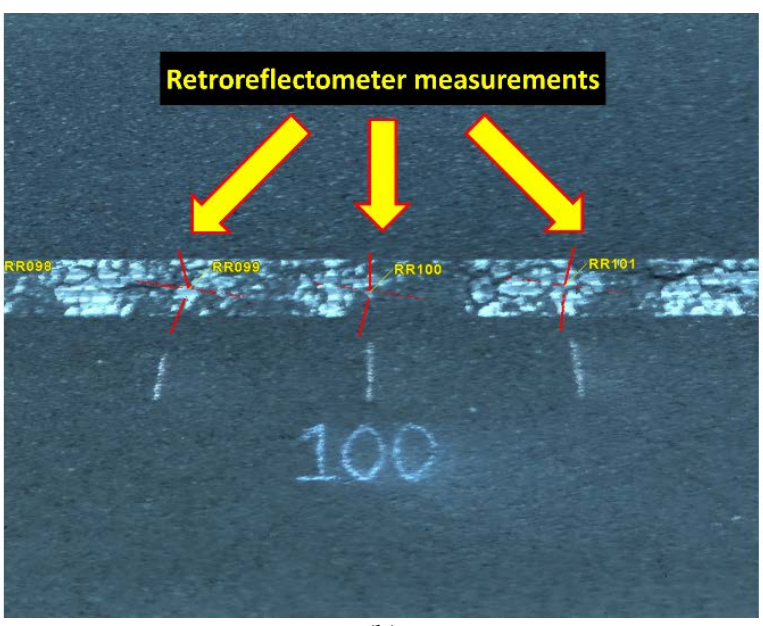

(b)

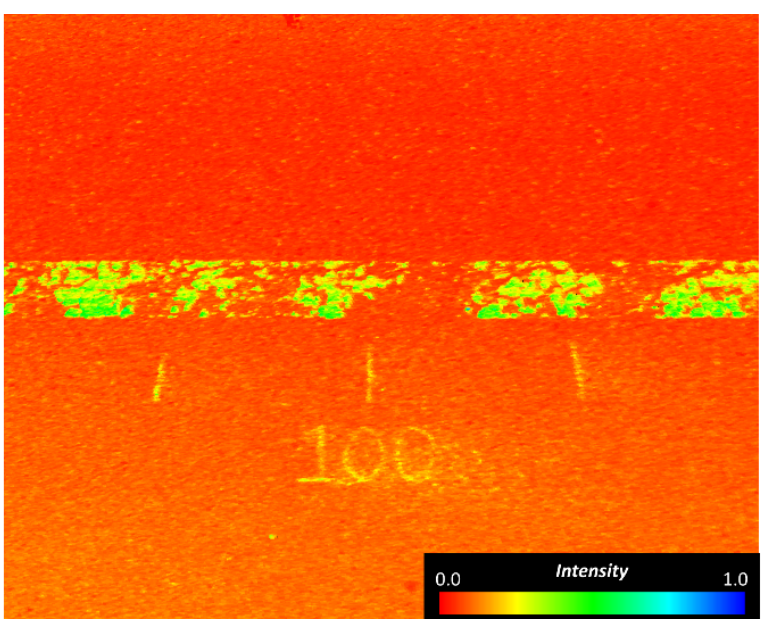

(c)

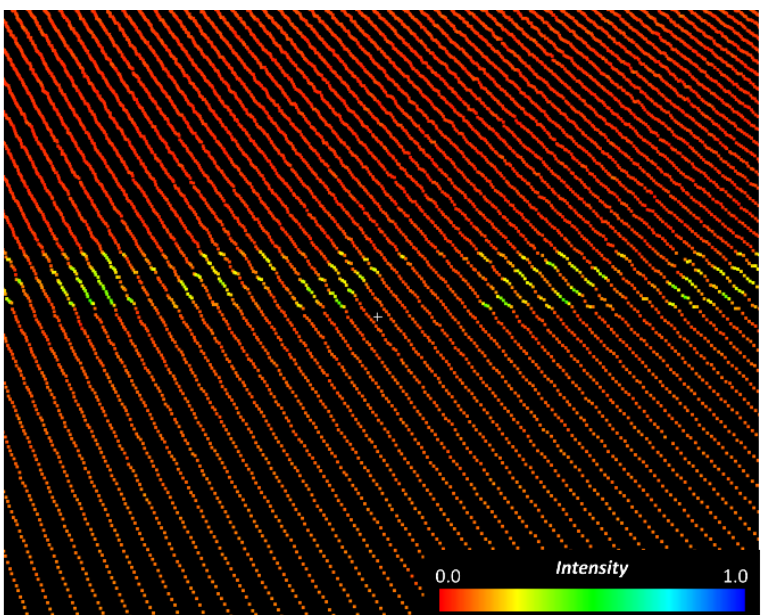

(d)

Figure 3. Examples of (a) photograph of a section of worn road marking, (b) RGB of the TLS point cloud and retroreflectometer samples, (c) Intensity of the TLS point cloud, (d) Intensity of the MLS point cloud. 


\section{ANALYSES}

\subsection{Radiometric calibration regression}

Olsen et al. (2018) performed radiometric calibration to convert the intensity values of the MLS point clouds to retroreflectivity measurements based on an empirical regression with the handheld retroreflectometer. However, the radiometric calibration model including both the sampling technique and regression function cannot be directly used for TLS data since it is specific to the Leica Pegasus: Two. One primary reason is that compared against a relatively consistent point density obtained with a MLS scan in the driving direction due to a consistent driving speed, the point density of a TLS scan varies significantly in a relatively short range (Figure 4) based on proximity to the scanner.

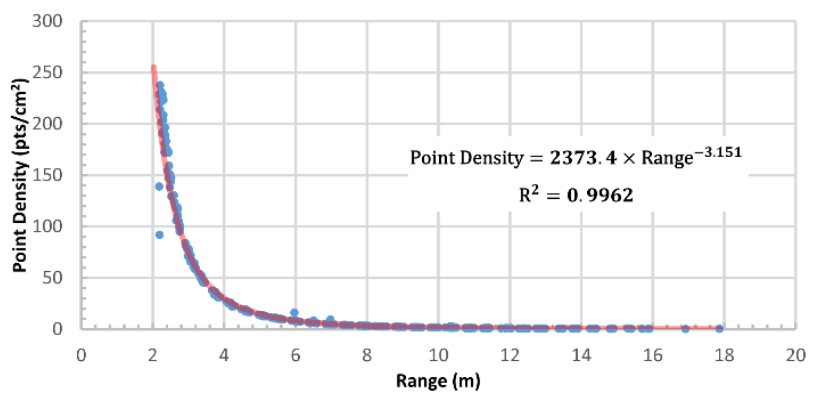

Figure 4. Correlation between range and point density at the sampling points on the stripe.

Although a clear trend is shown that can potentially be used to correct for the impacts of point density, unfortunately, such a correction is not straightforward to apply in practice, due to the missing information between scanlines (or rows) where the marking quality may vary substantially. Although the spacing varies considerably up to $10 \mathrm{~m}$ from the scanner, after $10 \mathrm{~m}$, the curve in Figure 4 flattens and the spacing becomes very sparse (Figure 5). Additionally, the spacing within a scanline becomes substantially different than the spacing within a scan row where points are closer together. The point cloud still shows significant differences in terms of point distribution.
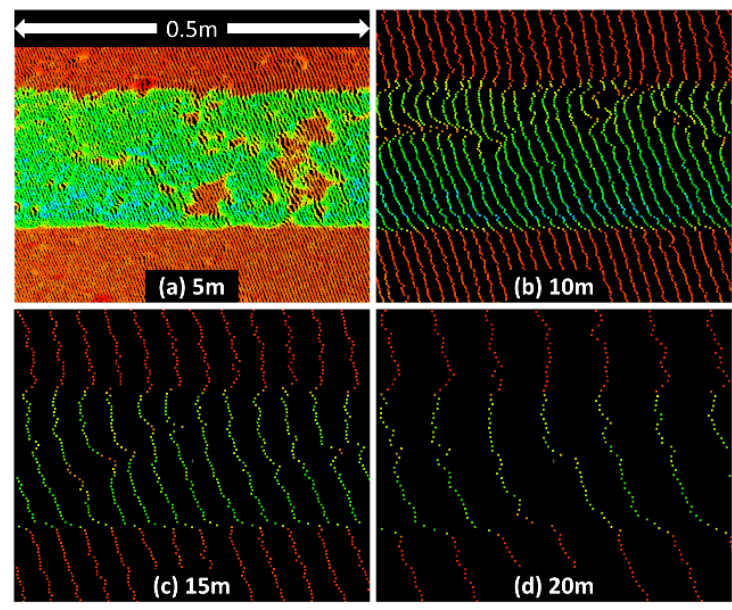

Figure 5. Examples of varying point densities within a point cloud from a single scan at (a) $5 \mathrm{~m}$, (b) $10 \mathrm{~m}$, (c) $15 \mathrm{~m}$, (d) $20 \mathrm{~m}$ from the scanner (Note that four different parts of the stripe are shown in this figure at the same scale).
To counter such uneven distribution of TLS data, we propose a weighting strategy for the radiometric calibration. Based on the principle of how a retroreflectometer operates of aggregating a reading of retroreflectivity over a window, we utilize a footprint of the same dimensions as the handheld retroreflectometer to aggregate the intensity from a set of points, and correlate that with the retroreflectivity readings rather than applying an intensity correction to each individual point. The retroreflectomter has both an active footprint (illumination field) to be illuminated by the light source in the instrument, as well as a passive window (observation field) for capturing the light that is returned (DELTA Light \& Optics, 2016). To further account for the point distribution, we rasterize the point cloud within the footprint (active/passive window) and compute the coverage ratio (ratio between the number of covered grid cells and the total number of grid cells within that footprint) as the weight in the following model regression (Figure 6). A grid cell size of 0.001 m was used.

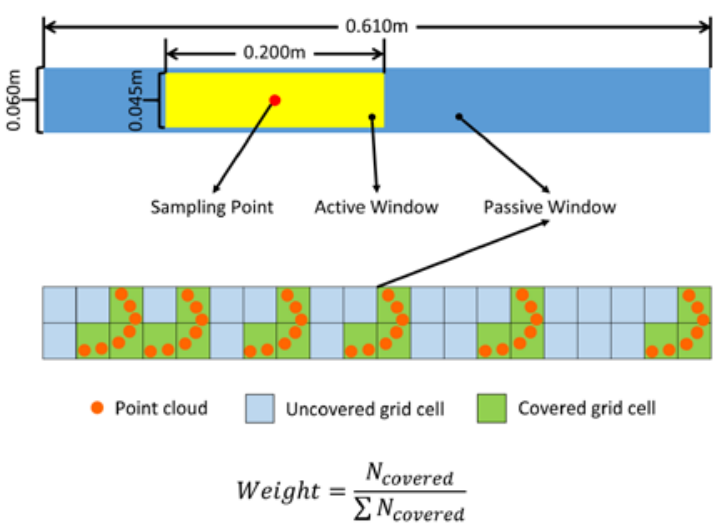

Figure 6. Schemetic illustration of the footprint of the retroreflectometer and the weighting scheme taking the passive window as an example.

Che et al. (in press) used the upper $10^{\text {th }}$ percentile of the intensity values in an active window for with a power function for model regression for MLS data. In this proposed work for TLS data, a linear model was chosen since the power function was similar over the range of retroreflectivity values tested. We also compared regression results using an average intensity in the active window, $10^{\text {th }}$ percentile of intensity values in the active window, and average intensity in the passive window. The average intensity value within the passive window outperformed the other two sampling strategies. These results are presented in Figure 7 , where a linear regression was applied with the aforementioned weighting strategy both using points extracted from the windows for each scan separately (Figure 7(a)) and points extracted from the windows for with all of the scans combined (Figure 7(b)) for comparison.

Although the R-squared value for the linear regression using the points from each single scan individually is slightly lower than using points from all the scans combined when sampling, it is noticeable that the sample size of the former scenario is significantly smaller than the latter one. Nevertheless, the resulting equations are very similar and only differ by less than $0.3 \mathrm{mcd} / \mathrm{l} \mathrm{x} / \mathrm{m}^{2}$ over the ranges of intensity tested. 


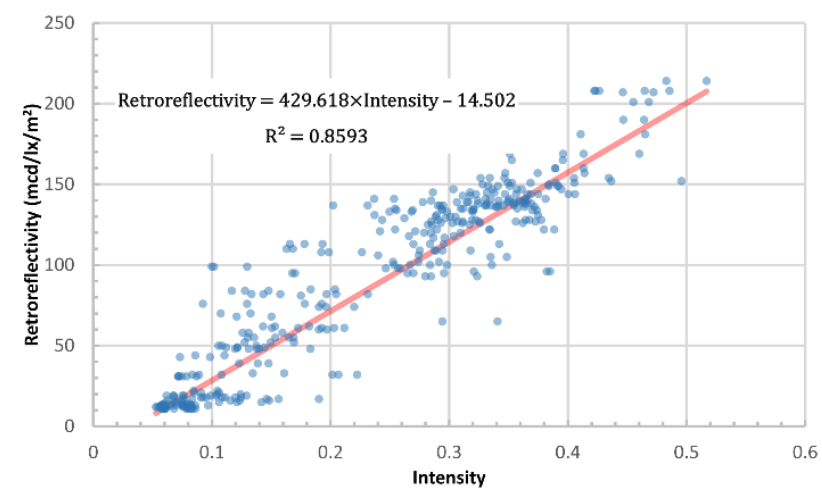

(a)

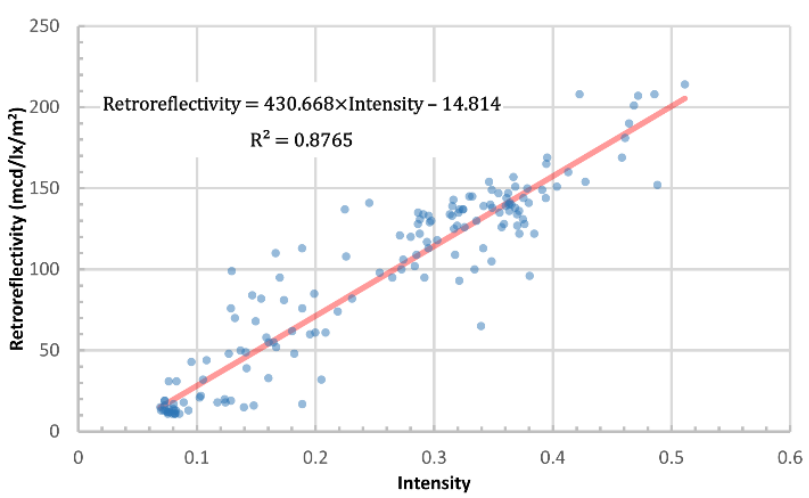

(b)

Figure 7. Linear regression results using (a) points from each single scan within the passive window treated separately; (b) average of points from all the scans combined within the passive window.

\subsection{Investigation of Intensity Corrections}

We investigated a further intensity correction in terms of range and incidence angle, which tend to be two of the most common and substantial corrections applied in radiometric calibration. The road surface is typically close to a planar surface; hence, once the instrument height is known, the incidence angle would be highly correlated to the range (Figure 8 ). The incidence angle can be estimated through the theoretical relationship derived based on geometry in Figure 8 (red line) with the assumption of a planar surface for the road. A more refined estimate of the incidence angle can be determined by computing a normal vector within a local neighbourhood rather than estimating a planar surface (blue dots in Figure 8). Notice that when the range is large ( $\sim 10 \mathrm{~m})$, the estimated incidence angle computed based on a normal determined within a local neighbourhood rather than an estimated planar surface deviates from this theoretical correlation as a result of additional uncertainty in the normal estimation occurring from the lower point density (Figure 4). Thus, integration of multiple scans are usually recommended to cover the area of interest and to ensure the point density and the reliability of the information extracted from the point clouds.

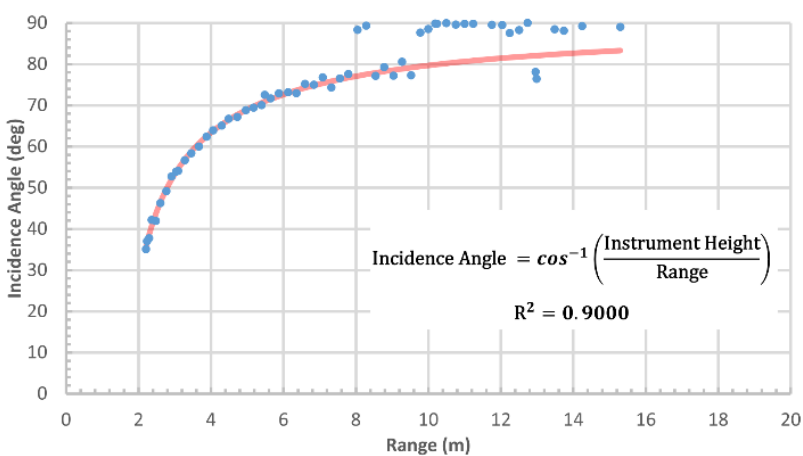

Figure 8. Example of correlation between range and incidence angle for the points on the stripe where the instrument height was $1.79 \mathrm{~m}$.

Such a strong correlation between range and incidence angle created by the TLS scanning geometry could enable one to develop an empirical model with a single variable to correct the intensity. In this case, we consider range as the variable to explore the potential correction. In particular, retroreflective materials are designed to reflect light back in the direction of the source; hence, the actual angle of incidence would be closer to $0^{\circ}$ compared with the estimated angle of incidence based solely on geometry without considering material type.

To evaluate the variance of intensity with range, we first sample a number of points at different ranges lying on the pavement surface (asphalt). A very strong correlation can be seen between range and the intensity (Figure 9(a)) for the pavement surface; hence a simple correction (e.g., logarithmic function) can be applied to normalize the intensity values within this range. For the retroreflective road marking, we tested the points within a small bin of retroreflectivity readings between 140 and $150 \mathrm{mcd} / \mathrm{lx} / \mathrm{m}^{2}$ Figure 9(b)). However, there is no distinguishable correlation between intensity and retroreflectivity for the points lying on the road markings at the ranges evaluated and the trends can fluctuate when evaluated across a smaller window of ranges. Also it should be noted that the intensity can sometimes increase at further ranges from the scanner (also at a larger nominal incidence angle), which does not follow most of the theoretical models utilized for correcting intensity. The reason this occurs is that glass beads are usually mixed in with the paint to ensure the reflectivity of the markings. These beads reflect light directly and do not follow the Lambertian reflection model, which serves as a basic assumption for many radiometric calibration models. As a further complication, it is essentially unpredictable whether a laser beam will actually hit a glass bead in the markings, especially for the worn ones where beads are highly variable. As a result, it is difficult to apply one single correction for all the points lying on the markings to further estimate the retroreflectivity of the markings. Such reasons also explain the noise shown in the correlation between the intensity and retroreflectivity (Figure 7). Therefore, in this work, we will use the uncorrected intensity to estimate the retroreflectivity given that 1) when combining scans together, the average will be controlled by the closest scan based on the point density (Figure 4) and 2) the fact that a very strong correlation is obtained without the correction. 


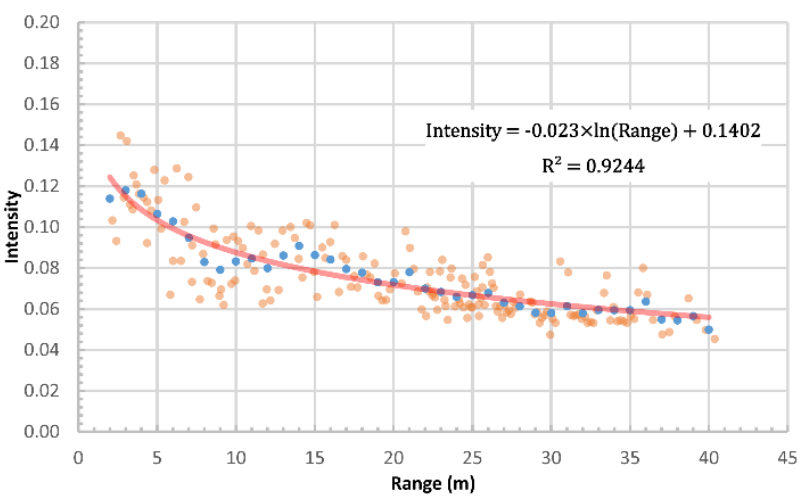

(a)

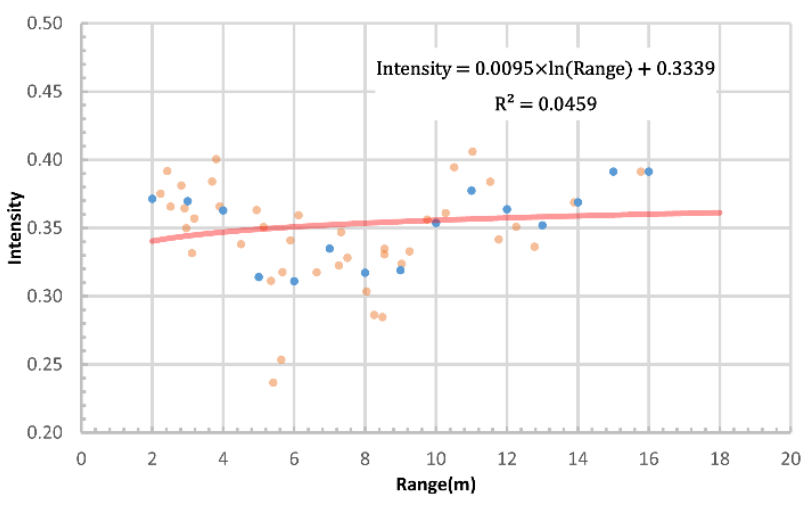

(b)

Figure 9. Correlation between range and intensity for points lying on: (a) pavement (asphalt); (b) road markings with a retroreflectivity reading between 140 and $150 \mathrm{mcd} / \mathrm{lx} / \mathrm{m}^{2}$. Orange markers represent individual points and blue points represent the average for each $1 \mathrm{~m}$ range bin.

\subsection{Comparative analysis}

To assess the accuracy of the proposed radiometric calibration approach, we compare the results to both the ground truth retroreflectometer measurements and the results of radiometric calibration for MLS data based on Olsen et al. (2018). For each lane, the retroreflectivity is first estimated from each pass and then averaged. The errors for different datasets and radiometric calibration approaches can be calculated and further summarized statistically (Table 1). The results show that for TLS data with all of the scans combined, using the passive window and averaging the intensity values within this footprint provides the best result. However, the results are fairly consistent between the different footprint sampling strategies in terms of RMSE compared to the variability of measurements from a handheld retroreflectometer.

For context in Table 1, for retroreflective measurements on the methyl methacrylate (MMA) thermoplastic stripes, according to ASTM1710-11, the reproducibility standard deviation is 22.9 $\mathrm{mcd} / \mathrm{lx} / \mathrm{m}^{2}$ while the repeatability standard deviation is 14.4 $\mathrm{mcd} / \mathrm{lx} / \mathrm{m}^{2}$. Because reproducibility considers the ability of independently achieving the same results with a different system, operator, calibration and conditions whereas repeatability considers the ability of achieving the same results with repeated measurements with the same device in similar conditions.
Table 1. Statistical summary of the errors in estimating retroreflectivity using TLS and MLS point clouds compared with the handheld retroreflectometer. (unit: $\mathrm{mcd} / \mathrm{lx} / \mathrm{m}^{2}$ )

\begin{tabular}{c|cccc}
\hline & Min & Max & Mean & RMSE \\
\hline $\begin{array}{c}\text { MLS } \\
\text { left lane }\end{array}$ & -58.6 & 77.8 & -3.3 & 21.8 \\
\hline $\begin{array}{c}\text { MLS } \\
\text { right lane }\end{array}$ & -66.0 & 60.8 & -7.5 & 24.3 \\
\hline $\begin{array}{c}\text { TLS } \\
\text { active window 10\% tile }\end{array}$ & -64.2 & 90.6 & -2.4 & 23.9 \\
\hline $\begin{array}{c}\text { TLS } \\
\text { active window average }\end{array}$ & -72.9 & 65.6 & -1.3 & 20.3 \\
\hline $\begin{array}{c}\text { TLS } \\
\text { passive window average }\end{array}$ & -58.1 & 66.4 & -1.0 & 19.4 \\
\hline
\end{tabular}

By further investigating the impact of the marking condition on the performance of different radiometric calibration models (Figure 10), it can be concluded that the proposed method using TLS data fits the ground truth significantly better than the MLS data (both left and right lanes) in estimating the retroreflectivity. There are two primary reasons why the proposed approach using TLS data is more accurate than our previous approach for MLS data. First, the TLS in this case has a higher spatial resolution such that it can capture more details in terms of reflectance (e.g., intensity) across the worn stripe. Additionally, for TLS data, the validation is performed using the same dataset that was used to develop the radiometric calibration model, while the model for MLS was developed from a completely separate dataset. Thus, the TLS model may be over fit to this specific dataset.

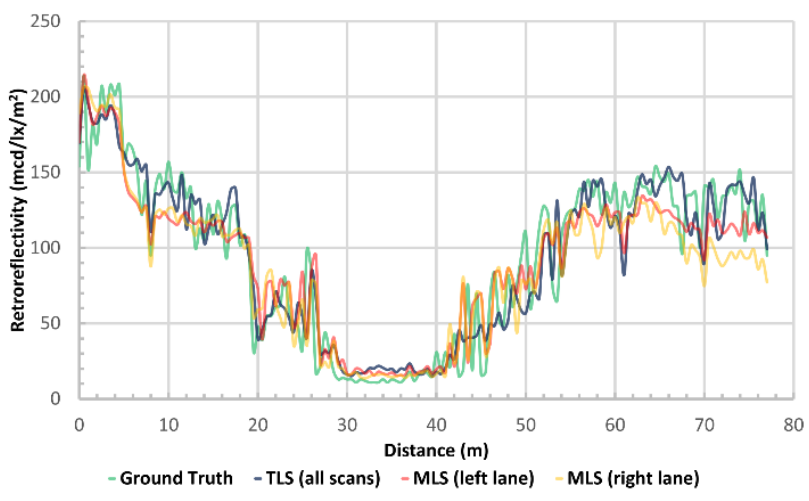

Figure 10. Comparison between retroreflectometer readings, and retroreflectivity estimation from TLS scans and MLS data.

In Section 3.1, selecting points from all of the scans combined or with each scan individually can result in a difference in evaluating the retroreflectivity. As a result, we investigate differences between the scan located at the centre of the area of interest (Scan \#5), and compare it against the results using aggregated points from all the scans combined. The comparison shows that these two strategies behave similarly. This occurs because even though all of the scans are combined, the points considered in the averaging within a given window will be predominantly from the closest scan. 


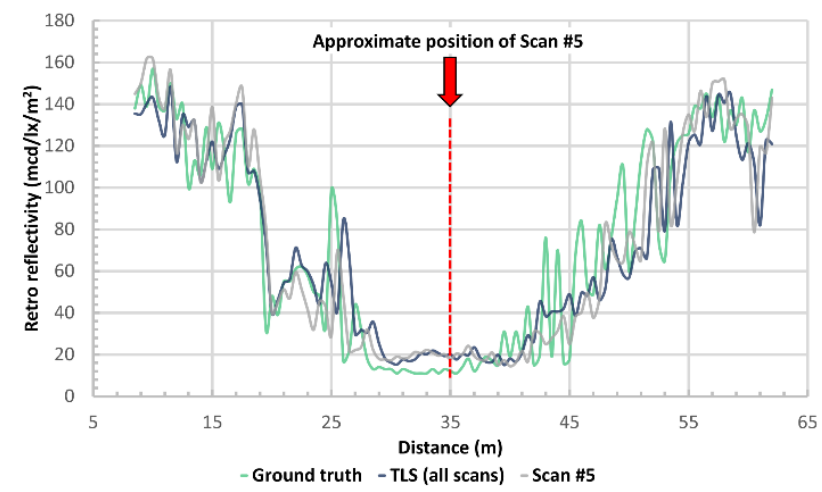

Figure 11. Comparison of retroreflectivity estimation using the points from a single scan and all scans combined.

\section{CONCLUSIONS}

In this study, the Leica P40 TLS was found to be effective for pavement marking evaluation and to correlate well with handheld retroreflectometer measurements as well as those obtained with MLS. Corrections for range and angle of incidence (nominal) remain elusive but also are less applicable in this particular application given the material properties to reflect light back in the direction of the source.

With any empirical study such as this, results should be used with caution. Outside the range of parameters tested (e.g., retroreflectiivty, range, nominal angle of incidence, etc.), the results may deviate from the correlations found in this study. Additionally, because the same dataset is used for generating the radiometric calibration model as well as the verification, the result can be overfit to this dataset. We will address this issue by using reference targets and testing more data in our future work. Moreover, intensity behaviour is different with other scanner models by different, or even the same manufacture. Manufacturers also sometimes apply internal corrections, so we are working with manufacturers to obtain the raw intensity signals. Furthermore, the model was developed for lower ranges of retroreflectivity. Should higher levels of retroreflectivity be of interest, the model should be developed up to the point of saturation for the scanner.

\section{ACKNOWLEDGEMENTS}

This research was funded in part, by Oregon DOT (SPR-799) and the National Science Foundation (Award CMMI-1351487). We appreciate the support of Joel Fry, Lloyd Bestdoe, Dan Wright, and Jon Lazarus, from Oregon DOT who assisted with the retroreflectivity measurements, mobile lidar scanning, mobile lidar data processing, and research coordination, respectively. Oregon State University students Chase Simpson, Kory Kellum, Nick Wilson, Katherine Shaefer, Marian Jamieson, and Nick Forfinski assisted with data collection. The authors also appreciate the support of Leica Geosystems and David Evans and Associates who provided hardware and software used in this research.

\section{REFERENCES}

Ai, C. and Tsai, Y.J., 2016. An automated sign retroreflectivity condition evaluation methodology using mobile LIDAR and computer vision. Transportation Research Part C: Emerging Technologies, 63, pp.96-113.

Carlson, P., Storey, B., Poorsartep, M., Stevens, C., Ettelman, B., Lindheimer, T.E., et al., 2017. Advancing innovative high-speed remote-sensing highway infrastructure assessment using emerging technologies: technical report, Texas A\&M Transportation Institute.

Che, E., Olsen, M.J., Parrish, C.E., and Jung, J. (in press). Pavement Marking Retroreflectivity Estimation and Evaluation using Mobile Lidar Data, Photogrammetric Engineering \& Remote Sensing.

DELTA Light \& Optics, 2016. LTL-X Mark II Retrometer: UserManual. https://roadsensors.madebydelta.com/products/ltlx-mark-ii-retroreflectometer/.

Guan, H., Li, J., Cao, S., and Yu, Y. 2016. Use of mobile LiDAR in road information inventory: A review, International Journal of Image and Data Fusion, 7, pp. 219-242, 2016.

Guan, H., Li, J., Yu, Y., Wang, C., Chapman, M. and Yang, B., 2014. Using mobile laser scanning data for automated extraction of road markings. ISPRS Journal of Photogrammetry and Remote Sensing, 87, pp.93-107.

Jaakkola, A., Hyyppä, J., Hyyppä, H. and Kukko, A., 2008. Retrieval algorithms for road surface modelling using laser-based mobile mapping. Sensors, 8(9), pp.5238-5249.

Jung, J., Che, E., Olsen, M.J. and Parrish, C., 2019. Efficient and robust lane marking extraction from mobile lidar point clouds. ISPRS Journal of Photogrammetry and Remote Sensing, 147, pp.1-18.

Kashani, A.G., Olsen, M.J., Parrish, C.E., and Wilson, N., 2015. A review of LiDAR radiometric processing: From ad hoc intensity correction to rigorous radiometric calibration, Sensors, 15, pp. 28099-28128.

Olsen, M.J., Roe, G.V., Glennie, C., Persi, F., Reedy M., Hurwitz, D., et al. 2013. Guidelines for the Use of Mobile LIDAR in Transportation Applications, NCHRP Report \#748.

Olsen, M.J., Parrish, C.E., Che, E., Jung, J., and Greenwood, J., 2018. Lidar for maintenance of pavement reflective markings and retroreflective signs. Vol I. Reflective Markings, Oregon DOT SPR-799 Final Report.

Toth, C., Paska, E., and Brzezinska, D., 2008. Using road pavement markings as ground control for LiDAR data," International Archives of Photogrammetry, Remote Sensing and Spatial Information Sciences, 37, pp. 189-195.

Zhang, H., Li, J., Cheng, M., and Wang, C., 2016. Rapid Inspection of pavement markings using mobile lidar point clouds, International Archives of the Photogrammetry, Remote Sensing \& Spatial Information Sciences, 41. 